\title{
A mayor prescripción de antidepresivos menor tasa de suicidio
}

Association between antidepressant prescribing and suicide in Australia, 1991-2000: trend analysis. Wayne D. Hall, Andrea Mant, Philip B. Mitchell, et al. BMJ. 2003;326(7397):1008.

\section{Objetivo}

Examinar la asociación entre la tendencia prescribir antidepresivos (ADP) y tasas de suicidio en Australia entre 1991 y 2000.

\section{Diseño}

Estudio ecológico cuasi-experimental ${ }^{\star}$ que analizó datos recolectados prospectivamente sobre suicidios y prescripción de ADP provenientes del Bureau Australiano de Estadísticas, de la industria farmacéutica australiana y del IMS Health Australia.

Lugar

Australia.

\section{Pacientes}

Hombres y mujeres desde los 15 años en adelante, divididos en ocho grupos $(15-24,25-34,35-44,45-54,55-64,65-74,75-84, \geq 85$ años).

\section{Medición de resultados principales}

Tendencias en la proporción de suicidios asociada a la prescripción de ADP(correlación de Spearman)* combinando información sobre consumo de alcohol, tasas de desempleo y métodos de suicidio. Resultados principales

Si bien la tasa nacional de suicidios no disminuyó significativamente, la incidencia en adultos mayores decreció, aumentando en adultos jóvenes (en su mayoría varones). Se comprobó que a mayor exposición a los ADP, hubo una significativa mayor declinación de la tasa de suicidio en Hombres ( $r s=-0,91$; IC95\% -0,57 a -0,98) $y$ en mujeres ( $r s=-0,76 ;-0,12$ a $-0,95)$ especialmente en grupos de mayor edad (ver figuras 1 y 2 ).

Figuras 1 y 2: cambios en la tasa de suicidios/100.000 personas (período 1986-90 y 1996-2000) en ordenadas, según el nivel de exposición a antidepresivos (DDD/1.000 personas/día) * en abcisas,
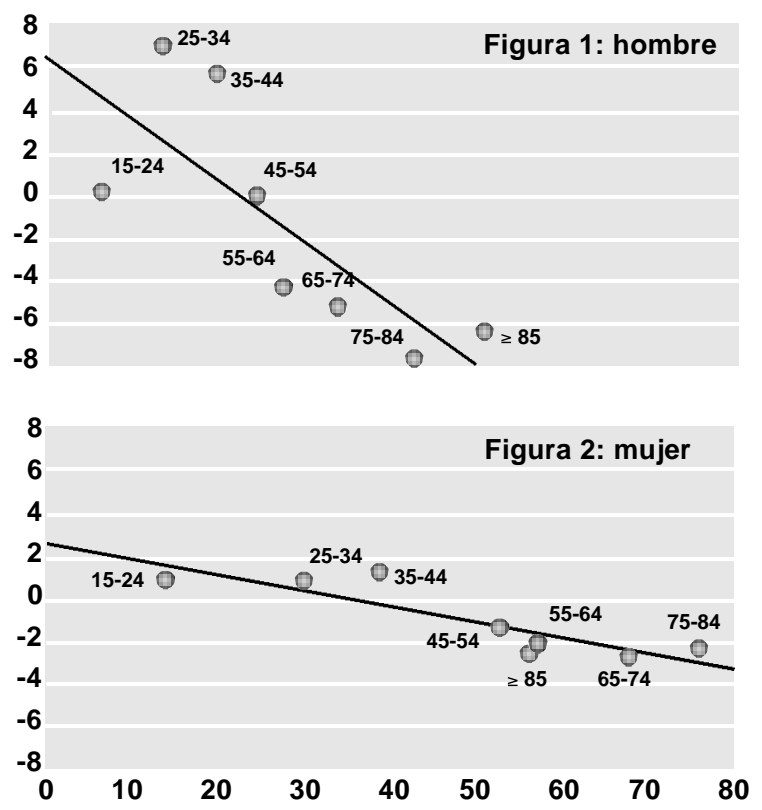

por grupos de edad.

\section{Conclusiones}

Los cambios en las tasas de suicidio y la exposición a los ADPen Australia entre 1991- 2000 están significativa e inversamente asociados. Este efecto fue más aparente en los pacientes mayores en quienes la tasa de suicidio disminuyó significativamente en asociación con la administración de ADP

\section{Comentario}

Lo observado en este estudio coincide con lo reportado de otros países como Suecia y Hungría ${ }^{1-3}$. El incremento en la administración de ADPy su mantenimiento puede constituirse en indicador poderoso del mejoramiento del manejo de la depresión. La prescripción de ADPinhibidores de la recaptación de serotonina (IRSS) en la practica general podría entonces producir un importante beneficio en la salud mental de la población. Sin embargo, al estudiar factores ecológi$\cos ^{*}$ y no individuales, las correlaciones entre tasa de suicidio y prescripción de ADP no indican necesariamente que la indicación de ADP haya reducido la tasa de suicidio en pacientes deprimidos.

La tendencia en los factores de riesgo suicida como el desempleo y el consumo de alcohol pueden explicar la disminución de suicidios ya que el consumo de alcohol declinó en el comienzo de los noventa para luego mantenerse fijo ${ }^{4}$ y el desempleo creció tanto para los mayores como para los jóvenes ${ }^{5}$. La disminución de la tasa de suicidio en el grupo etáreo mayor podría explicarse por el mejoramiento de la calidad de vida, aunque esto no se reflejó en las encuestas de calidad de vida ${ }^{6}$. No se encontraron diferencias en los métodos de suicidio, descartándose que la disminución de suicidios sea consecuencia de campañas de educación.

Coincido con los autores en cuanto a los beneficios de la administración de ADP en pacientes deprimidos ya que, al mejorar el cuadro el cuadro depresivo, disminuye el riesgo suicida. Es importante la detección precoz de los síntomas depresivos, así como la indagación de las ideas de suicidio, desmitificando la idea de que el profesional podría inducir el suicidio al interrogar al paciente acerca de fantasías o planes suicidas. La desocupación, el alcoholismo y los an- tecedentes de enfermedad mental son factores de riesgo a ser indagados cuidadosamente.Asimismo la detección de cuadros depresivos con ideación suicida implicará no sólo la administración de ADPsino también la indicación de psicoterapia (está comprobado que la combinación de ambas es más efectiva que la administración de ADPsolamente) así como el asesoramiento y la participación de la familia. Los nuevos ADP presentan reacciones adversas menos frecuentes y de menor intensidad que los IMAO y los ADPtricíclicos, permitiendo una mayor adherencia de los pacientes al tratamiento y un manejo menos complicado para el médico.

Es probable que la disminución de la tasa de suicidio en adultos mayores (grupo etáreo de mayor riesgo) en Australia haya disminuido en la medida en que el sistema previsional funciona de manera muy diferente al de nuestro país, permitiendo que la gente mayor esté protegida por el Estado. El lugar que ocupan los mayores en una sociedad depende de la valoración que cada cultura tiene de sus mayores: en nuestro país al no estar insertos en el sistema productivo son desvalorizados hasta el punto de convertirse en una variable de ajuste económico. Si bien no disponemos de cifras oficiales en la Argentina la tasa de suicidio o al menos las tentativas de suicidio se han incrementado en función de la crisis económica.

Conclusión del comentador: El presente estudio refuerza el rol de los ADP, encontrándose el médico en posición privilegiada para la detección precoz de cuadros depresivos y riesgo suicida en sus pacientes; debiendo actuar con premura, citando a la familia y administrando ADP como primer medida terapéutica, previa, de ser necesaria, a la derivación al especialista.

Dr. Daniel Kitanik [ Psiquiatra del Área de Salud Mental. Unidad de Medicina Familiar y Preventiva. Hospital Italiano de Buenos Aires. ]

*Ver glosario

\section{Referencias}

1. Isacsson G. Suicide preventiona medical breakthrough? Acta Psychiatr Scand 2000; 102: 113-117

2. Carlsten A, Waern M, Ekedahl A, Ranstram J. Antidepressant medication and suicide in Sweden. Pharmacoepidemiol Drug Saf 2001; 10: 525-530

3. Rihmer Z. Can better recognition and treatment of depression reduce suicide rates? Abrief review. Eur Psychiatry 2001; 16: 406-409

3. Rihmer Z. Can better recognition and treatment of depression reduce suicide rates? Abrief review. Eur Psychiatry 2001; 16: 406-409
4. Catalano P, Chikritzhs T, Stockwell T, Webb M, Rohlin C-J, Dietze P. Trends in per capita alcohol consumption in Australia, 1990/91-1998/99. Perth: National Drug Research Institute, 2001. (Nation-

4. Catalano P, Chikritzhs T, Stockwell T, Webb M, Rohlin C-J, Dietze P. Trends in per capita alcohol consumption in Australia,
al Alcohol Indicators Bulletin No 4.)
5. Morrell S, Page A, Taylor R. Unemployment and youth suicide. Economic and Labour Relations Review 2001; 12: 4-17.

5. Morrell S, Page A, Taylor R. Unemployment and youth suicide. Economic and Labour Relations Review 2001; 12: 4-17.
6. Heady B. Old age is not downhill: the satisfactions and well-being of older Australians. Aust J Ageing 1999; 18(suppl): 32-37. 